\title{
Three-Dimensional Visualization of Murine Cardiac Tissue Using FIB-SEM Segmentation Techniques
}

\author{
George Wetzel $^{1}$, Jamil J. Clarke ${ }^{2}$, Joan Hudson ${ }^{1}$ \\ ${ }^{1}$ Clemson University Advanced Materials Research Laboratory, Anderson, SC, USA \\ ${ }^{2}$ Hitachi High Technologies America, Gaithersburg, MD, USA
}

Traditional techniques for producing three-dimensional renderings of a sample include serial sectioning/imaging and electron tomography. Both of these techniques can be utilized to generate high resolution renderings, but are met with certain limitations, including strict sample requirements, limited tilt range, and electron beam-sample interactions [1].

Focused ion beam (FIB) instruments are used extensively in the semiconductor and materials science sectors for site-specific analysis, but are normal not applied to biological type materials due to inherent sample damage caused by the ion beam. Combined focused ion beam and scanning electron microscope (FIB-SEM) instruments also utilize a high energy $\mathrm{Ga}^{+}$ion beam to sputter material from a sample, but a built in SEM gun allows for damage-free observation of the specimen. Furthermore, many FIB-SEMs allow for simultaneous milling and observation for live time fabrication of a sample. Ion beam conditions, including accelerating voltage and aperture size, can be changed to adjust sputtering rate to accommodate a wide variety of samples, from soft polymers to hardened tool steels.

Here, we demonstrate the use of a FIB-SEM as a method to acquire data for 3D reconstruction. We have taken an embedded mouse heart and run it through the Mill \& Monitor feature of the Hitachi nanoDUE'T NB5000 FIB-SEM. Mill \& Monitor is a fully automated program that uses the ion beam to cut vertical slices into the sample, after each of which an image of the milled surface is imaged using the electron beam. The ExB filtering system allows for acquisition of images using a mix of both backscattered and secondary electron signals for images of sufficient contrast even on smooth surfaces.

The sample used was mouse heart tissue originally intended for microtomy and TEM analysis. Staining with osmium tetroxide using the OTO procedure [2] meant that the sample exhibited sufficient contrast for SEM imaging, and embedding entirely in resin ensured the structure would maintain structural integrity upon exposure to the ion beam. To help alleviate charge-up of the embedding media, the specimen block was sputter coated with a thin layer of platinum.

Upon loading the sample into the microscope, a high energy ion beam was quickly scanned across the sample to etch the surface and reveal any underlying structures. A potential location of interest was identified via SEM observation. Mill \& Monitor was then carried at that location, and the resulting images were collated into a $3 \mathrm{D}$ rendering.

A challenge of creating a 3D model from a stack of 2D images involves calculating the correct Zdistance of each image. Mill \& Monitor allows the user to specify the exact thickness of each slice, and stability of the stage ensures that fine pitch is maintained even when cutting ultrathin sections. For our demonstration, we cut a total of 159 slices at 0.094 um thick each, for a total cutting depth of about $15 \mathrm{um}$. Furthermore, because of the fine pitch, we were able to generate high detail images along the YZ direction of the $3 \mathrm{D}$ rendering, which is perpendicular to the original face that was imaged. 
[1]Bruce F. McEwen, Michael Marko, J. Histochem Cytochem (2001) 49:553

[2]Arnold Seligman et al, J Cell Biol. (1966) 30(2) 424-432

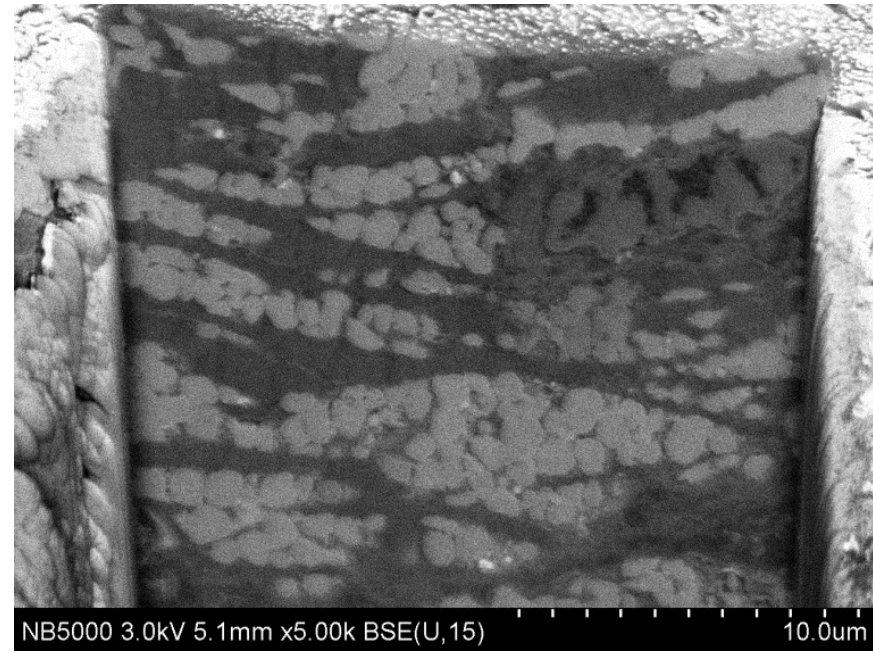

Figure 1. Single view after milling. Nucleus (upper right quadrant) and multiple mitochondira (white spheres) are visible. ExB filter allows for detection of compositional data, resulting in enhanced contrast on smooth surfaces.

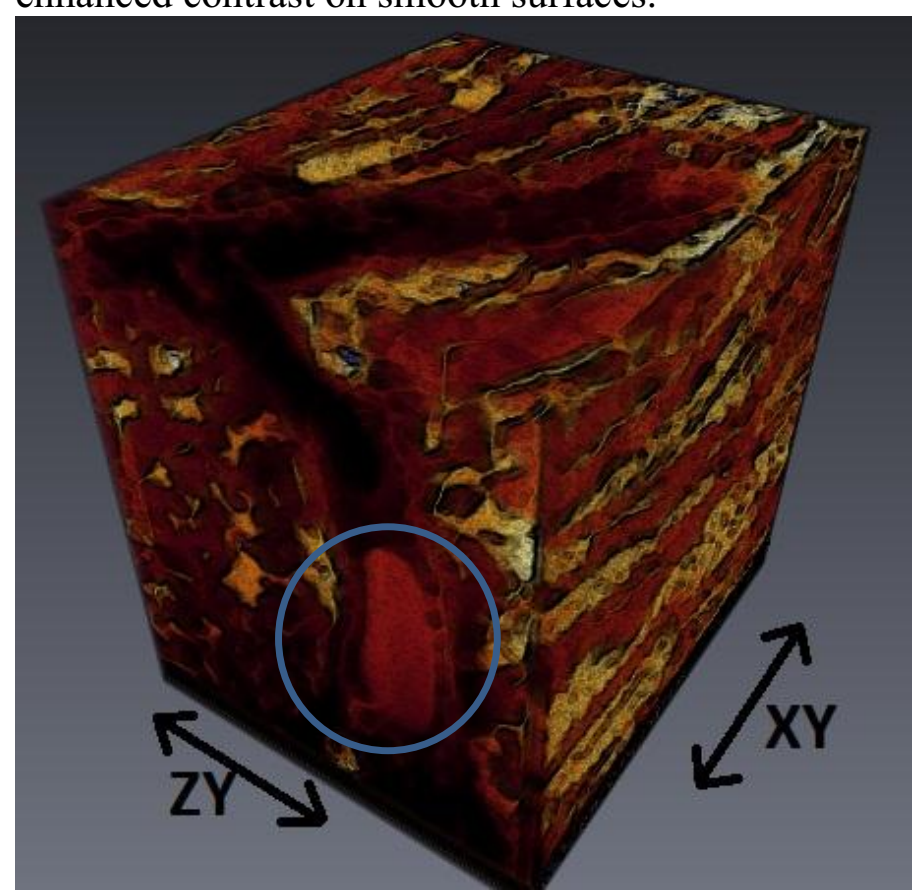

Figure 2. 3D rendering of sectioned mouse heart assembled from sliced data set. Original plane of observation (e.g. Figure 1) along XY axis. ZY direction can be viewed after assembling into model. This allows observation of features that may otherwise be missed if only examined from one direction (e.g. Red blood cell circled in blue)

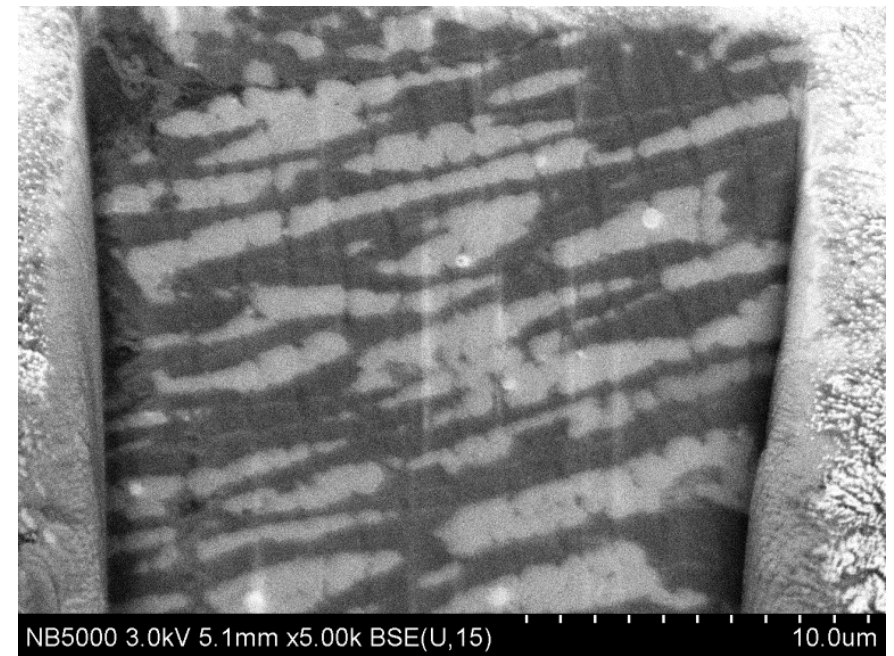

Figure 2. FIB-SEM still exhibits fine detail capabilities. Note the presence of intercalated discs. 